\title{
ADESÁO À TERAPIA ANTIRRETROVIRAL EM MULHERES: INFLUÊNCIA DO PERFIL CLÍNICO E COMPORTAMENTAL DE SAÚDE
}

\author{
ADHERENCE TO ANTIRETROVIRAL THERAPY IN WOMEN: \\ INFLUENCE OF THE CLINICAL AND BEHAVIORAL HEALTH PROFILE
}

\author{
ADHERENCIA A LA TERAPIA ANTIRRETROVIRAL EN MUJERES: \\ INFLUENCIA DEL PERFIL DE SALUD CLÍNICO Y DEL \\ COMPORTAMIENTO
}

\author{
Juliana Da Rocha Cabral* \\ Danielle Chianca De Andrade Moraes** \\ Luciana Da Rocha Cabral ${ }^{* * *}$ \\ Daniela De Aquino Freire**** \\ Fátima Maria Da Silva Abrão***** \\ Regina Celia De Oliveira $* * * * * *$
}

\begin{abstract}
RESUMO
Objetivo: Analisar a adesão à terapia antirretroviral em mulheres vivendo com HIV a partir do perfil clínico e comportamental de saúde. Material e Método: Estudo epidemiológico, descritivo, transversal, com abordagem quantitativa. A população foi composta por mulheres que vivem com HIV que são assistidas pelos Serviços de Assistência Especializada (SAEs) em HIV em cinco municípios localizados na I Gerência Regional de Saúde (GERES) de Pernambuco, Brasil. A amostragem do estudo ocorreu por intermédio do método não probabilístico, um sorteio aleatório, com o intuito de atingir um representação de $30 \%$ do total de serviços adscritos na I GERES. Os participantes do estudo compuseram a amostra, também a partir de um método não probabilístico por conveniência, por tanto, 184 mulheres vivendo com HIV fizeram parte do estudo. A coleta de dados ocorreu no interstício compreendido entre junho a agosto de 2018. Utilizou-se o Cuestionario Evaluación de la Adhesión al Tratamiento Antirretroviral (CEAT-VIH) para mensurar a adesão das participantes. Resultados:
\end{abstract}

*Enfermeira. Mestre em Enfermagem. Programa Associado de Pós-Graduação da Universidade de Pernambuco (UPE), Recife, Pernambuco, Brasil. ORCID: https://orcid.org/0000-0003-3827-996X Email: jucabral06@hotmail.com Autora correspondente **Enfermeira. Doutora em Enfermagem. Programa Associado de Pós-Graduação da Universidade de Pernambuco (UPE), Recife, Pernambuco, Brasil. ORCID: https://orcid.org/0000-0003-4979-6145 Email: dani_chianca@hotmail.com

***Enfermeira. Mestranda em Enfermagem. Programa Associado de Pós-Graduação da Universidade de Pernambuco (UPE), Recife, Pernambuco, Brasil. ORCID: https://orcid.org/0000-0002-6396-3897 Email: lucabral06@hotmail.com

****Enfermeira. Mestre em Enfermagem. Programa Associado de Pós-Graduação da Universidade de Pernambuco (UPE), Recife, Pernambuco, Brasil. ORCID: https://orcid.org/0000-0002-6708-5139 Email: daniela_3439@hotmail.com

*****Enfermeira. Doutora em Enfermagem. Faculdade de Enfermagem Nossa Senhora das Graças da Universidade de Pernambuco, Recife, Pernambuco, Brasil. ORCID: https://orcid.org/0000-0003-3254-2851 Email: fatima.abrao@upe.br

****** Doutora em Enfermagem. Faculdade de Enfermagem Nossa Senhora das Graças da Universidade de Pernambuco. Recife, Pernambuco, Brasil. ORCID: https://orcid.org/0000-0002-6559-5872 Email: regina.oliveira@upe.br 
Apresentaram maior prevalência para uma boa adesão: o grupo que se enquadra no nível de instrução superior incompleto/completo apresenta 1,69 vezes mais prevalência de adesão à Terapia Antirretroviral (TARV); mulheres não ativas sexualmente apresentam $103 \%$ de chances a mais para uma boa adesão; e aquelas em que cujo prontuário não consta informação de abandono à TARV: 94\%. Conclusão: À luz dos desafios atuais associados à adesão à TARV, tem-se evidenciado a necessidade da elaboração de estratégias de cunho social destinadas a mulheres vivendo com HIV a fim de promover melhor qualidade de vida a essa população.

Palavras-chave: HIV; Mulheres; Adesão à medicação.

\section{ABSTRACT}

Objective: To analyze adherence to Antiretroviral Therapy (ART) among women living with HIV from a clinical and behavioral health profile. Material and Methods: Epidemiological, descriptive, cross-sectional study, with a quantitative approach. The population consisted of women living with HIV, who are assisted by the Specialized HIV Care Services (SAEs) in five municipalities located in the I Regional Health Management Center (GERES) of the State of Pernambuco, Brazil. The sampling of the study was carried out through a non-probabilistic method (random drawing) in order to reach a representation of $30 \%$ of the total number of services enrolled in the I GERES. The sample was also set up using a non-probabilistic method by convenience, so 184 women living with HIV were part of the study. Data collection occurred between June and August 2018. The Cuestionario para la Evaluación de la Adhesión al Tratamiento Antirretroviral (CEAT-VIH) was used to measure the adherence of the participants. Results: The group that falls in the incomplete/complete higher education level has 1.69 times more prevalence of adherence to ART, non-sexually active women present $103 \%$ chances for good adherence; and those whose medical record does not contain information on abandonment of ART: 94\%. Conclusion: Considering the current challenges associated with adherence to ART, there is a clear need to develop social strategies aimed at women living with HIV in order to promote better quality of life for this population.

Key words: HIV; Women; Medication Adherence.

\section{RESUMEN}

Objetivo: Analizar la adherencia a la terapia antirretroviral en mujeres viviendo con VIH desde el perfil clínico y de salud conductual. Material y Método: Estudio epidemiológico, descriptivo, transversal con abordaje cuantitativo. La población estuvo conformada por mujeres viviendo con VIH que son atendidas por Servicios de Atención Especializada (SAE) en VIH en cinco municipios ubicados en la I Gerencia Regional de Salud (GERES) de Pernambuco, Brasil. El estudio se muestreó mediante el método no probabilístico, un sorteo aleatorio, con el objetivo de alcanzar una representación del 30\% del total de servicios registrados en el I GERES. Los participantes del estudio compusieron la muestra, también utilizando un método no probabilístico por conveniencia, por lo que 184 mujeres que viven con el VIH fueron parte del estudio. La recolección de datos se realizó en el intersticio entre junio y agosto de 2018. Se utilizó el Cuestionario Evaluación de la Adhesión al Tratamiento Antirretroviral (CEAT-HIV) para medir la adherencia de los participantes. Resultados: Hubo una mayor prevalencia de buena adherencia: el grupo que se ajusta al nivel de educación superior incompleta/ completa tiene 1,69 veces más prevalencia de adherencia a la Terapia Antirretroviral (TAR); las mujeres no sexualmente activas tienen un $103 \%$ más de posibilidades de una buena adherencia; y aquellos cuyos registros médicos no contienen información sobre abandono del TAR: 94\%. Conclusión: A la luz de los desafíos actuales asociados a la adherencia al TAR, se ha resaltado la necesidad de desarrollar estrategias sociales dirigidas a las mujeres que viven con el VIH con el fin de promover una mejor calidad de vida para esta población.

Palabras clave: HIV; Mujeres; Adherencia a la medicación. 


\section{INTRODUÇÃO}

Ao logo dos anos, tem-se acompanhado um crescimento na incidência do HIV em mulheres e, com isso, há maior estigma relacionado à infecção na população feminina, o que confere maior solidão ao processo de elaboração da sua convivência com o vírus $^{(1)}$. Estatísticas do ano 2021 revelam que a cada semana, cerca de 5 mil mulheres entre 15 e 24 anos de idade são infectadas pelo HIV, em nível global ${ }^{(2)}$.

Ouso da Terapia Antirretroviral (TARV) de forma eficaz e diária reduz significativamente a morbidade e mortalidade pela AIDS, além de contribuir com o controle das infecçôes oportunístas, melhoria da qualidade de vida, redução da transmissão do HIV pela supressão virológica e diminuição do risco de resistência viral, permitindo maiores possibilidades terapêuticas ${ }^{(3-5)}$.

A adesão à TARV geralmente é influenciada pela associação de diversos fatores/variáveis, inerentes ou não ao sujeito submetido ao tratamento. Por isso, a avaliação dessa adeão torna-se indispensável no que concerne a criação de estratégias de tratamento em pessoas que vivem com o HIV (PVHIV) ${ }^{(6)}$.

Mulheres vivendo com o HIV que não aderem à TARV devem ser priorizadas nas estratégias de controle da doença, como garantia do fortalecimento dos direitos sexuais e reprodutivos femininos. Dessa forma, os profissionais de saúde devem trabalhar de maniera integrada para o desenvolvimento de açôes que corroborem com à adesão, além do monitoramento e acompanhamento clínico desse público alvo ${ }^{(7)}$.

Nesse contexto, a enfermagem, que ocupa maior corpo clínico dos serviços de saúde, exerce um papel central na potencialização de ações-chaves para melhoria da saúde e o bem-estar das mulheres, por meio de atividades educativas preventivas, apoio à adesão medicamentosa e discussóes acerca de comorbidades e estilos de vida. Ademais, os enfermeiros também podem utilizar sua experiência para defender estratégias necessárias para gerenciar as barreiras para uma adesão satisfatória ${ }^{(8,9)}$.

Ressalta a necessidade de estudos contínuos sobre esse tema, pois é por meio do questionamento e da análise dos entraves para o tratamento, que se encontram soluçóes para tornar o enfrentamento do HIV um pouco menos difícil para as mulheres ${ }^{(7)}$.

Inserido nesse contexto, sabe-se que a adesão à TARV é a ação fundamental para a mudança no cenário atual da doença. Sendo assim, o presente estudo tem como objetivo analisar a adesão à terapia antirretroviral a partir do perfil clínico e comportamental de saúde em mulheres vivendo com HIV.

O conhecimento da relação do perfil clínico e comportamental de saúde na adesão à terapia antirretroviral em mulheres vivendo com HIV é importante para que os serviços especializados em HIV melhor conduzam seus planos de cuidado para o fortalecimento da adesão medicamentosa.

\section{MATERIAL E MÉTODOS}

Trata-se de um estudo epidemiológico, descritivo, transversal, com abordagem quantitativa, desenvolvido em cinco municípios localizados na I Gerência Regional de Saúde (GERES) de Pernambuco, Brasil.

O estado selecionado para a pesquisa é dividido em XII GERES, contemplando 37 serviços de assistência integral, gratuita e especializada em HIV, vinculadas ao Sistema Único de Saúde (SUS), para o atendimento do público adulto. Atualmente a I GERES corresponde a maior densidade técnológica e de quantitavo de serviços em saúde e conta com 17 Serviços de Assistência Especializada em HIV (SAEs). Destaca-se que a I GERES fora escolhida por ser a região de saúde onde a Universidade vinculada ao estudo encontra-se localizada.

A população foi composta por mulheres que vivem com HIV que são assistidas pelos SAEs em HIV da I GERES do estado de Pernambuco. A amostragem do estudo ocorreu por intermédio do método não probabilístico, um sorteio aleatório, com o intuito de atingir um representação de 30\% do total de serviços adscritos na I GERES. Assim, cinco serviços de diferentes municípios foram contemplados no estudo, a saber: Recife $(A=98)$, Gravatá $(B=10)$, Igarassu $(C=14)$, Vitória de Santo Antão (D=28) e Olinda (E=34). Os participantes do estudo compuseram a amostra, também a partir de um método não probabilístico, por conveniência. Por tanto, 184 mulheres vivendo com HIV fizeram parte do estudo.

Para os critérios de elegibilidade, foram incluídos mulheres vivendo com HIV que realizavam tratamento com a TARV por período igual ou superior há 6 meses, possuíam idade igual ou maior que 
18 anos e realizam atendimento multiprofissional com a equipe do serviço em estudo. Posteriormente, foram excluídas as mulheres grávidas e que apresentaram registro em prontuário de comprometimento cognitivo que interferisse na comunicação e no entendimento das questóes referentes à realização da entrevista.

A coleta de dados ocorreu no interstício compreendido entre junho a agosto de 2018. As mulheres foram convidadas a participar do estudo enquanto aguardavam, em sala de espera, o atendimento com a equipe multidisciplinar, nos dias da coleta ou que compareceram à farmácia do serviço para pegar à TARV e demais medicamentos. Foram realizadas entrevistas individuais, em salas dos próprios ambulatórios que proporcionavam privacidade para o entrevistado e entrevistador. Todas as participantes receberam informaçôes pertinentes ao estudo e demostraram concordância em participar a partir da assinatura do Termo de Consentimento Livre e Esclarecido.

Desenvolveu-se um questionário estruturado, elaborado pela própria equipe de pesquisa, contendo as variáveis independentes a partir dos dados sociodemográficos (sexo, idade, renda, escolaridade, estado civil e vínculo empregatício), comportamentais de saúde e clínico (prática da atividade física, tempo de diagnóstico e tratamento, uso de preservativo, via de contágio do HIV, histórico de infecções oportunistas e registro de adesão irregular em prontuário).

Além disso, utilizou-se um instrumento autoaplicável, a fim de se captar a variável dependente, descrita pela adesão, investigada por meio do instrumento "Cuestionario para la Evaluación de la Adhesión al Tratamiento Antiretroviral” (CEATVIH), desenvolvido na Espanha, considerado válido e confiável para medir a adesão ao tratamento da TARV. O instrumento é composto por 20 questóes, avaliado em um escore de 17 a 89 pontos, sendo classificada em três níveis: adesão estrita (boa), quando o resultado da soma foi maior ou igual a 80 pontos, o que significa uma adesão acima de 85\%; adesão regular (dificuldade com o cumprimento), apresentando somatório final entre 75 e 79 pontos, representando 50 a $85 \%$ de adesão à terapia; e uma adesão insuficiente (baixa), se o resultado da soma foi menor que 74 pontos, representando menos de $50 \%$ de adesão à TARV ${ }^{(10)}$.

Nesse estudo, as mulheres vivendo com HIV que apresentaram percentual de adesão maior ou igual a $85 \%$ foram classificadas como boa adesão, em vez de adesão estrita, e os que apresentaram percentual de adesão menor que $85 \%$ serão classificados como baixa adesão. Essa adaptação foi feita para facilitar as análises bivariadas, facilitando a associação e consequentemente a identificação ou não de significância estatística ${ }^{(11)}$.

Para análise dos dados foi construído um banco no programa EPI INFO, versão 3.5.1, em que foi realizada a digitação e validação do banco de dados com dupla digitação para posterior comparação e correção dos valores divergentes. Após a digitação do banco, os dados foram exportados para o software SPSS, versão 18 para realização da análise estatística.

Para avaliar a influência do perfil clínico e comportamento de saúde e no CEAT-VIH foram construídas as tabelas de contingência e aplicado o teste Qui-quadrado para independência. Nos casos em que as suposiçôes do teste não foram satisfeitas aplicou-se o teste Exato de Fisher. Todas as conclusôes foram tiradas considerando o nível de significância de 5\%.

Para análise multivariada foram incluídos os fatores que apresentaram significância de até 20\% na análise bivariada. Sendo assim, aplicou-se o modelo de Poisson com variância robusta para avaliação do risco de boa adesão pelos pacientes avaliados. Ainda, foram calculados os intervalos de confiança para a razão da prevalência e o teste de Wald na comparação dos riscos para a boa adesão pelo CEAT-VIH entre os níveis dos fatores avaliados.

Realizou-se um recorte da dissertação intitulada "Adesão e expectativa de autoeficácia à terapia antirretroviral e associação com a qualidade da assistência de enfermagem".

O estudo obedeceu à Resolução No 466/2012 do Conselho Nacional de Saúde, tendo sido aprovado no Comitê de Ética em Pesquisa do Complexo Hospitalar da Universidade de Pernambuco sob parecer 2.545.008.

\section{RESULTADOS}

Perfil sociodemográfico das participantes (Tabela 1): Os extremos etários variaram de 18 a 70 anos e média de 42,2 anos. Observa-se, ainda 
o baixo grau de instrução com a escolaridade até o $1^{\circ}$ grau com maior representatividade $(44,0 \%)$. Chama atenção o elevado quantitativo de mulheres sem parceria afetiva sexual fixa, $112(60,9 \%)$ declararam ser solteria, viúva ou divorciada. No que concerne a renda familiar, 91 (49,4\%) afirmaram renda familiar menor que um salário mínimo.

Perfil clínico e comportamental das avaliadas (Tabela 2): Foi possível identificar que a maioria das participantes não realiza atividade física $(71,7 \%)$. Quanto ao tempo de diagnóstico e tratamento para o HIV, percebe-se a maior prevalência para mais de cinco anos de tratamento (54,9\%). O uso irregular do preservativo fora mencionado por 32 mulheres $(17,4 \%)$. Já em relação ao conhecimento e via de infecção pelo HIV, a maior parte das participantes refere ser a relação sexual desprotegida como via de contaminação $(95,3 \%)$. O teste de comparação de proporção foi significativo em todos os fatores avaliados ( $\mathrm{p}<0,001)$, indicando que o perfil descrito é significativamente o mais frequente entre os pacientes avaliados.

Ainda na Tabela 2, percebe o menor registro em prontuário, por parte dos profissionais de saúde, sobre a adesão irregular. Observou-se em 58 (31,5\%) dos prontuários das mulheres entrevistadas consta a informação de adesão irregular à medicação. Entretanto, tal fato difere do que fora observado na avaliação autorrelatada das mulheres vivendo com HIV acerca da adesão à TARV, medido pelo instrumento CEAT-VIH.

Mulheres vivendo com HIV e adesão à TARV: Na Tabela 3 é apresentada as estatísticas descritivas dos escores que avaliam a classificação do CEATVIH. A adesão regular/ruim representou destaque $(65,2 \%)$ da avaliação, os extremos dos escores variaram de 47 a 84 pontos. O teste de comparação de proporção foi significativo $(\mathrm{p}<0,001)$, indicando que a prevalência de adesão regular/ruim é relevantemente maior no grupo avaliado.

Adesão à TARV e variáveis clínicas e comportamentais de saúde: $\mathrm{Na}$ Tabela 4 verifica-se a distribuição da classificação do CEAT-VIH segundo as variáveis clínicas e comportamentais de saúde. Houve maior prevalência de boa adesão pelo CEAT-VIH no grupo de participantes que afirmaram realizar alguma atividade física $(34,6 \%)$, bem como, aqueles com mais de 5 a 10 anos de diagnóstico e tratamento $(36,0 ; 36,4 \%$, respectivamente).

Tabela 1. Distribuição das mulheres vivendo com HIV, segundo características sociodemográficas. Recife, PE, Brasil, 2018.

\begin{tabular}{llccc}
\hline Fator avaliado & & n & $\%$ & p-valor $^{\mathbf{1}}$ \\
\hline Idade (Media de 42,2 ańos) & 18 a 28 & 15 & 8,2 & $<0,001$ \\
& 29 a 39 & 63 & 34,2 & \\
& 40 a 60 & 98 & 53,3 & \\
& Maior do que 60 & 8 & 4,3 & \\
\hline Grau de instrução & Nenhum & 6 & 3,3 & $<0,001$ \\
& 1' grau comp/incompl & 81 & 44 & \\
& 2o grau comp/incompl & 80 & 43,5 & \\
& Superior comp/incompl & 17 & 9,2 & \\
\hline Estado civil & Casada/com companheira & 71 & 38,8 & $<0,001$ \\
& Solteira & 84 & 45,9 & \\
& Viúva & 6 & 3,3 & \\
& Separada/divorciada & 22 & 12 & \\
\hline Renda Familiar* & Menor que um salário mínimo & 91 & 49,4 & $<0,001$ \\
& Entre 1 a 2 salários mínimos & 57 & 31 & \\
& Mais que 3 salários mínimos & 36 & 19,6 & \\
\hline
\end{tabular}

${ }^{1}$ p-valor do teste Qui-quadrado para comparação de proporção (se p-valor $<0,05$ as proporçôes diferem significativamente).

*Valor do salário mínimo na época de realização do estudo: BRL 880,00. 
Tabela 2. Distribuição das mulheres vivendo com HIV, segundo características clínicas e comportamentais de saúde. Recife, PE, Brasil, 2018.

\begin{tabular}{|c|c|c|c|c|}
\hline Fator avaliado & & $\mathbf{n}$ & $\%$ & p-valor ${ }^{1}$ \\
\hline \multirow[t]{2}{*}{ Realiza atividade física } & Sim & 52 & 28,3 & $<0,001$ \\
\hline & Não & 132 & 71,7 & \\
\hline \multirow[t]{4}{*}{ Tempo de diagnóstico } & Menos de 1 ano & 20 & 10,9 & $<0,001$ \\
\hline & De 1 a 5 anos & 63 & 34,2 & \\
\hline & Mais de 5 anos a 10 anos & 50 & 27,2 & \\
\hline & Mais de 10 anos & 51 & 27,7 & \\
\hline \multirow[t]{4}{*}{ Tempo de tratamento HIV } & Menos de 1 ano & 119 & 64,7 & $<0,001$ \\
\hline & De 1 a 5 anos & 6 & 3,3 & \\
\hline & Mais de 5 anos a 10 anos & 26 & 14,1 & \\
\hline & Mais de 10 anos & 33 & 17,9 & \\
\hline \multirow[t]{4}{*}{ Utiliza preservativo } & Sim & 119 & 64,7 & $<0,001$ \\
\hline & Não & 6 & 3,3 & \\
\hline & Às vezes & 26 & 14,1 & \\
\hline & Não possui relação sexual & 33 & 17,9 & \\
\hline \multirow[t]{2}{*}{ Sabe como adquiriu HIV } & Sim & 129 & 70,1 & $<0,001$ \\
\hline & Não & 55 & 29,9 & \\
\hline \multirow[t]{3}{*}{ Como adquiriu } & Relação sexual & 123 & 95,3 & $<0,001$ \\
\hline & Acidente com pérfuro-cortante & 4 & 3,1 & \\
\hline & Transfussão sanguínea & 2 & 1,6 & \\
\hline \multirow{2}{*}{$\begin{array}{l}\text { Registro de adesão irregular à TARV em } \\
\text { prontuário }\end{array}$} & Sim & 58 & 31,5 & $<0,001$ \\
\hline & Não & 126 & 68,5 & \\
\hline
\end{tabular}

${ }^{1}$ p-valor do teste Qui-quadrado para comparação de proporção (se p-valor $<0,05$ as proporçôes diferem significativamente).

Tabela 3. Distribuição das mulheres vivendo com HIV, segundo adesão à TARV, conforme escores do CEAT-VIH. Recife, PE, Brasil, 2018.

\begin{tabular}{lccc}
\hline Fator avaliado & $\mathbf{n}$ & $\mathbf{\%}$ & p-valor $^{\mathbf{1}}$ \\
\hline Boa adesão & 64 & 34,8 & $<0,001$ \\
Regular/ruim adesão & 120 & 65,2 & \\
\hline
\end{tabular}

${ }^{1}$ p-valor do teste Qui-quadrado para comparação de proporção (se p-valor $<0,05$ as proporçôes diferem significativamente).

As que alegaram não possuir relação sexual apresentaram a melhor adesão do grupo (51,5\%). Já as que afirmaram saber como adquiriu HIV também apresentaram prevalência maior para boa adesão $(34,1 \%)$. O mesmo foi observado naquelas que não apresentaram registro em prontuário de adesão irregular $(38,9 \%)$.
Modelo das variáveis para boa adesão à TARV em mulheres vivendo com HIV (Tabela 5): Mostramse as variáveis estudadas que foram inseridas na regressão de Poisson, para análise multivariada. Assim, somente o grau de instrução, uso do preservativo e registro de adesão irregular à TARV em prontuário, permaneceram no modelo final 
associadas à adesão. $\mathrm{O}$ grupo que se enquadra no nível de instrução superior incompleto/completo apresenta 1,69 vezes mais prevalência de adesão à TARV do que aqueles com $1^{\circ}$ grau completo/ incompleto. Ainda, todas essas razóes foram significativas indicando que a relação descrita é relevante.

Quanto ao utilizar preservativo, houve diferença significativa para uma boa adesão pelo CEAT-VIH entre o grupo de referência (que utiliza preservativo nas relaçóes sexuais) e com o grupo que não pratica relação sexual $(\mathrm{p}=0,002)$. Assim, urge dizer que as mulheres não ativas sexualmente apresentam 103\% de chances para uma boa adesão pelo CEAT-VIH do que o grupo que possui relação sexual protegida.

Em relação ao registro de adesão irregular à TARV, aquelas em que cujo prontuário não constam tal informação, apresentam $94 \%$ de condiçôes a mais para uma boa adesão pelo CEAT-VIH do que o grupo que não segue a tomada diária da TARV.

Tabela 4. Distribuição da classificação da adesão à TARV em mulheres vivendo com HIV pelo CEAT-VIH, segundo variáveis clínicas e comportamentais de saúde. Recife, PE, Brasil, 2018.

\begin{tabular}{|c|c|c|c|c|}
\hline \multirow[t]{2}{*}{ Fator avaliado } & & \multicolumn{2}{|c|}{ CEAT-VIH } & \multirow[b]{2}{*}{ p-valor ${ }^{1}$} \\
\hline & & Boa adesão & $\begin{array}{c}\text { Adesão } \\
\text { regular/baixa }\end{array}$ & \\
\hline \multirow[t]{2}{*}{ Realiza atividade física } & Sim & $18(34,6 \%)$ & $34(65,4 \%)$ & $0,642^{1}$ \\
\hline & Não & $41(31,1 \%)$ & $91(68,9 \%)$ & \\
\hline \multirow[t]{4}{*}{ Tempo de diagnóstico } & Menos de 1 ano & $4(20,0 \%)$ & $16(80,0 \%)$ & $0,626^{1}$ \\
\hline & De 1 a 5 anos & $21(33,3 \%)$ & $42(66,7 \%)$ & \\
\hline & Mais de 5 anos a 10 anos & $18(36,0 \%)$ & $32(64,0 \%)$ & \\
\hline & Mais de 10 anos & $16(31,4 \%)$ & $35(68,6 \%)$ & \\
\hline \multirow[t]{4}{*}{ Tempo de tratamento HIV } & Menos de 1 ano & $6(24,0 \%)$ & $19(76,0 \%)$ & $0,772^{1}$ \\
\hline & De 1 a 5 anos & $21(32,3 \%)$ & $44(67,7 \%)$ & \\
\hline & Mais de 5 anos a 10 anos & $16(36,4 \%)$ & $28(63,6 \%)$ & \\
\hline & Mais de 10 anos & $16(32,0 \%)$ & $34(68,0 \%)$ & \\
\hline \multirow[t]{4}{*}{ Utiliza preservativo } & Sim & $31(26,1 \%)$ & $88(73,9 \%)$ & $0,044^{2}$ \\
\hline & Não & $2(33,3 \%)$ & $4(66,7 \%)$ & \\
\hline & Às vezes & $9(34,6 \%)$ & $17(65,4 \%)$ & \\
\hline & Não possui relação sexual & $17(51,5 \%)$ & $16(48,5 \%)$ & \\
\hline \multirow[t]{2}{*}{ Sabe como adquiriu HIV } & Sim & $44(34,1 \%)$ & $85(65,9 \%)$ & $0,363^{1}$ \\
\hline & Não & $15(27,3 \%)$ & $40(72,7 \%)$ & \\
\hline \multirow[t]{3}{*}{ Como adquiriu } & Relação sexual & $42(34,1 \%)$ & $81(65,9 \%)$ & $0,513^{2}$ \\
\hline & Acidente com pérfuro-cortante & $2(50,0 \%)$ & $2(50,0 \%)$ & \\
\hline & Transfussão sanguínea & $0(0,0 \%)$ & $2(100,0 \%)$ & \\
\hline \multirow{2}{*}{$\begin{array}{l}\text { Registro de adesão irregular à TARV } \\
\text { em prontuário }\end{array}$} & Sim & $10(17,2 \%)$ & $48(82,8 \%)$ & $0,003^{1}$ \\
\hline & Não & $49(38,9 \%)$ & $77(61,1 \%)$ & \\
\hline
\end{tabular}

${ }^{1}$ p-valor do teste Qui-quadrado para independência (se p-valor $<0,05$ o fator avaliado influencia na classificação do CEAT-VIH). ${ }^{2}$ p-valor do teste Exato de Fisher. 
Tabela 5. Análise multivariada de modelo ajustado de Poisson para a boa adesão à TARV em mulheres vivendo com HIV pelo CEAT-VIH. Recife, PE, Brasil, 2018.

\begin{tabular}{|c|c|c|c|c|}
\hline Fator avaliado & & $\mathbf{R P}$ & IC(95\%) & p-valor ${ }^{1}$ \\
\hline \multirow[t]{3}{*}{ Grau de instrução } & $1^{\circ}$ grau comp/incompl & 1 & - & - \\
\hline & $2^{\circ}$ grau comp/incompl & 1,84 & $1,14-3,00$ & 0,013 \\
\hline & Superior comp/incompl & 2,69 & $1,53-4,73$ & 0,001 \\
\hline \multirow[t]{4}{*}{ Utiliza preservativo } & Sim & 1 & - & - \\
\hline & Não & 1,45 & $0,63-3,36$ & 0,376 \\
\hline & Às vezes & 1,17 & $0,68-2,01$ & 0,567 \\
\hline & Não possui relação sexual & 2,03 & $1,29-3,19$ & 0,002 \\
\hline \multirow{2}{*}{$\begin{array}{l}\text { Registro médico de adesão irregular à TARV } \\
\text { em prontuário }\end{array}$} & Sim & 1 & - & - \\
\hline & Não & 1,94 & $1,10-3,41$ & 0,022 \\
\hline
\end{tabular}

${ }^{1}$ p-valor da estatística de Wald (se -valor < 0,05 o risco para boa adesão é significativamente maior do que o grupo de referência).

\section{DISCUSSÃO}

Neste estudo foi usada uma estrutura para examinar adesão à terapia antirretroviral a partir do perfil clínico e comportamental de saúde em mulheres vivendo com HIV. Na análise, a relação da adesão baixa à TARV mostrou-se significativamente associadas a não prática do exercícios físico, ao tempo de diagnóstico e tratamento de um a cinco anos, ao uso do preservativo nas relaçóes sexuais, ao conhecimento da infecção do HIV pela relação sexual e a ausência de registro médico em prontuário de adesão irregular.

A caracterização sociodemográfica das participantes confirma o perfil da epidemia do HIV no Brasil, ao evidenciar o predomínio na faixa etária acima dos 40 anos, de baixa renda e exposição ao HIV por meio da transmissão sexual ${ }^{(1,12)}$.

Um estudo realizado nos Estados Unidos da América (EUA), com 2601 mulheres vivendo com o HIV também retrata epidemiologia semelhante, com média das idades em 40,5 anos e maior representatividade das solteiras. Contrapôs-se, no entanto, no grau de escolaridade, com identificação de menos de um terço da amostra com escolaridade menor que o ensino médio ${ }^{(4)}$. A diferença do desenvolvimento econômico brasileiro e americano pode justificar essa divergência.

Quanto ao comportamento sexual dessas mulheres, a observação do uso do preservativo nas relações sexuais, relatada por mais da metade das entrevistadas, é relevante, pois, tanto no nível individual quanto no coletivo, é o fator limitante da transmissão de infecções sexualmente transmissíveis e da reinfecção pelo HIV, para o parceiro sexual e para a população em geral $^{(13)}$.

A maior parte das mulheres deste estudo possuem mais de cinco anos de tempo de diagnóstico e tratamento. Tal achado, apresenta concordância com o perfil atual das PVHIV em acompanhamento ambulatorial. O maior tempo de convívio com a infecção pode facilitar o conhecimento acerca da importânica da adesão medicamentosa, para supressão virológica do $\mathrm{HIV}^{(14)}$. Por outro lado, a cronificação do tratamento dificulta a adesão ao longo dos anos, pela probabilidade da resistência aos medicamentos e pela necessidade de tomada diária dos remédios ${ }^{(15)}$.

Os achados que versam sobre a verificação da adesão medicamentosa, a partir da identificação do registro da equipe médica do serviço, demostraram menor representatividade da adesão irregular em prontuário. Contudo, a análise dos escores do CEAT-VIH contrasta-se com esses dados, pois, apenas, pouco mais de um terço das entrevistadas obtiveram pontuação para boa adesão.

Ao analisar estudos nacionais que versem sobre a adesão à TARV, observa-se similitude nos resultados da adesão insatisfatória ${ }^{(13,16)}$. Entretanto é necessário refletir que, pelo fato do HIV ser uma enfermidade crônica, a avaliação do grau de adesão ao tratamento medicamentoso do paciente não envolve um julgamento definitivo, o grau de adesão varia em qualquer período do tratamento, 
sendo influenciado por estilos e hábitos de vida. Dessa forma, a adesão deve ser constantemente estimulada pelos profissionais da saúde, tendo sempre em mente sua responsabilidade no sucesso ou fracasso da terapia ${ }^{(17)}$.

A principal causa de falha da TARV é a má adesão do paciente ao tratamento. A efetividade da TARV depende diretamente da adesão do paciente, e que o mesmo deve consumir pelo menos $85 \%$ dos medicamentos prescritos, para que a carga viral seja mantida indetectável ${ }^{(11)}$. Apesar de encontrar-se em evidência desde a década de 1980, ainda há lacunas no conhecimento em relação a influência do perfil clínico e comportamental de saúde na adesão à TARV em mulheres, por isso, faz-se necessários novos estudos na temática ${ }^{(18,19)}$.

É importante salientar que a adoção de hábitos saudáveis estão inseridos na amplitude do conceito de adesão. A atividade física, a exemplo, é indicada para o público em geral, principalmente, para as mulheres vivendo com HIV no que se refere à melhoria da capacidade funcional e prevenção de $\operatorname{agravos}^{(20)}$.

Uma pesquisa realizada no Nordeste do Brasil com PVHIV encontrou associação entre comportamento negativo para a saúde e a não realização de exercício físico, mostrando que esse fator é determinantes para a qualidade de vida dessa populaçáo. Assim, torna-se considerável a utilização de orientaçôes para o fortalecimento dessa prática no tratamento tradicional. Essa assistência gera positividade na $\mathrm{QV}$, e, consequentemente, na adesão medicamentosa ${ }^{(21)}$. A combinação entre inatividade física e adesão regular e baixa encontradas neste estudo, corrobora com essa pesquisa.

Apesar do uso do preservativo ser um hábito saudável e importante, não foi encontrada associação entre seu uso e a melhores taxas de adesão medicamentosa.

A baixa adesão é preocupante, uma vez que a adesão aos antirretrovirais é o foco principal para o sucesso terapêutico individual e pela possibilidade de reduzir as chances de infecção, minimizando incidência do HIV. Índices elevados de baixa adesão, significa que as mulheres vivendo com HIV apresentam dificuldades com à TARV e não estáo aderindo ao tratamento de forma segura e regular $^{(22)}$.

A diversidade de experiências analisadas aponta para a importância de estratégias de prevenção e de construção de um "viver com HIV" para mulheres que combinem intervençóes de cunho individual e coletiva com medidas estruturais mais amplas de redução das desigualdades sociais e de gênero ${ }^{(23)}$.

Por fim, conhecer a adesão à TARV em mulheres vivendo com HIV frente ao perfil clínico e comportamental de saúde torna-se essencial, visto que favorece o planejamento de políticas públicas que versem sobre a redução da transmissão do HIV, principalmente por cepas com resistência. Além disso, o enfermeiro exerce papel ímpar nesse contexto por realizar estratégias de orientaçóes e estimular a prática do autocuidado.

As limitaçóes deste estudo estáo relacionadas ao fato dos dados representarem apenas uma Gerência Regional de Saúde de um Estado brasileiro, podendo ter influência conforme características culturais e regionais. Por outro lado, o rigor metodológico utilizado e a validade interna do estudo traduz a confiabilidade dos dados.

\section{CONCLUSÃO}

O perfil clínico e comportamental de mulheres vivendo com HIV subsidia indicadores gerenciais fundamentais para realização de um diagnóstico situacional, planejamento de açóes e tomada de decisóes frente ao controle do HIV. No presente estudo, evidenciou-se importante prevalência da baixa adesáo à TARV. Observou-se, ainda, que a nível de escolaridade foi o principal fator que contribuiu para uma melhor adesão medicamentosa.

A partir do que fora encontrado, percebe-se a necessidade na implementaçáo de açóes a partir de atendimento humanizado e acolhedor, que possibilite uma escuta e considere as singularidades de cada indivíduo, a fim de minimizar o desestímulo para continuidade do tratamento e até mesmo fortalecer vínculo entre usuário-profissionaisserviço.

Espera-se que o resultado forneça subsídios para a reflexáo dos gestores e prossionais de saúde quanto ao emponderamento das mulheres vivendo com HIV acerca da sua condição de saúde-doença. Assim, aumentar o nível de conhecimento destas mulheres no processo do autocuidado, parece ser uma importante ferramenta de estímulo à adesão à TARV. 


\section{REFERÊNCIAS}

1. Villela WV, Barbosa RM. Trajetórias de mulheres vivendo com HIV/aids no Brasil. Avanços e permanências da resposta à epidemia. Ciênc saúde colet [Internet]. 2017 [citado $2019 \mathrm{dez} 21$ ]; 22(1): 87-96. Disponível em: https://www.scielosp.org/ article/csc/2017.v22n1/87-96/

2. World Health Organization (WHO). Estimativas epidemiológicas preliminares do UNAIDS 2021 [Internet]. [citado 2021 jun 9]. Disponível em: https://unaids.org.br/wp-content/uploads/2021/ 06/2020_11_19_UNAIDS_FactSheet_PORT_ Revisada-Final.pdf

3. Hogg RS, Eyawo O, Collins AB, Zhang W, Jabbari $S$, Hull MW, et al. Health-adjusted life expectancy in HIV-positive and HIV-negative men and women in British Columbia, Canada: a population-based observational cohort study. Lancet HIV [Internet]. 2017 [citado 2021 jun 9]; 4(6): e270-e2e6. Disponível em: https://doi.org/10.1016/S2352-3018 (17)30029-2

4. Benning L, Mantsios A, Kerrigan D, Coleman JS, Golub E, Blackstock O, et al. Examining adherence barriers among women with HIV to tailor outreach for long-acting injectable antiretroviral therapy. BMC womens health [Internet]. 2020 [citado 2021 jun 8]; 20(1): 152. Disponível em: https:// doi.org/10.1186/s12905-020-01011-8

5. Monteiro SS, Brigeiro M, Vilella WV, Mora C, Parker R. Desafios do tratamento como prevenção do HIV no Brasil: uma análise a partir da literatura sobre testagem. Ciênc saúde coletiva [Internet]. 2019 [citado 2019 dez 21]; 24(5): 1793-807. Disponível em: https://doi.org/10.1590/1413-81 232018245.16512017

6. Primeira MR, Santos WM, Paula CC, Padoin SMM. Qualidade de vida, adesáo e indicadores clínicos em pessoas vivendo com HIV. Acta Paul Enferm [Internet]. 2020 [citado 2021 jun 5]; 33: eAPE20190141. Disponível em: https://doi. org/10.37689/acta-ape/2020AO0141

7. Souza FBA, Sampaio ACL, Gomes MP, Silva GA, Silva ALB, Almeida EB. Changes in The Quotidian of Women Living With Hiv: Ambulatorial Analysis, Rio De Janeiro State, Brazil. Rev Fund Care [Internet]. 2019 [citado 2020 jan 12]; 11(5): 1260-65. Disponível em: http://dx.doi. org/10.9789/2175-5361.2019.v11i5.1260-1265

8. Xue Y, Smith JA, Spetz J. Primary Care Nurse Practitioners and Physicians in Low-Income and Rural Areas, 2010-2016. JAMA [Internet]. 2019 [citado 2021 jun 7]; 321(1): 102-105. Disponível em: https://doi.org/10.1001/jama.2018.17944.

9. Waldrop D, Irwin C, Nicholson WC, Lee CA,
Webel A, Fazeli PL, et al. The Intersection of Cognitive Ability and HIV: A Review of the State of the Nursing Science. J Assoc Nurses AIDS Care [Internet]. 2021 [citado 2021 jun 9]; 32(3): 306321. Disponível em: https://doi.org/10.1097/JNC .0000000000000232

10. Remor E, Milner-Moskovics J, Preussler G. Adaptação brasileira do "Cuestionario para la Evaluación de la Adhesión al Tratamiento Antiretroviral”. Rev Saúde Pública [Internet]. 2007 [citado 2020 jan 28]; 41(5): 685-94. Disponível em: https://doi. org/10.1590/S0034-89102006005000043

11. Bezabhe WM, Pharm B, Chalmers L, Bereznicki LR, Peterson GM. Adherence to Antiretroviral Therapy and Virologic Failure. A Meta-Analysis. Medicine [Internet]. 2016 [citado 2020 jan 28]; 95(15): 1-9. Disponível em: https://www.ncbi. nlm.nih.gov/pmc/articles/PMC4839839/pdf/ medi-95-e3361.pdf

12. Castrighini Cd, Reis RK, Neves LA, Melo ES, Gir E. Caracterización epidemiológica de personas que viven con VIH/sida. Rev Cubana Enferm [Internet]. 2019 [citado 2020 mar 1]; 35(1). Disponível em: http://revenfermeria.sld.cu/index. php/enf/article/view/1683

13. Candido PGG, Amador BM, Silva FF, Santos FS, Pinheiro LML, Oliveira Filho AB. Adherence to antiretroviral therapy among women living with HIV/AIDS in the interior of the Brazilian state of Pará: cross-sectional study. Sao Paulo Med J [Internet]. 2021 [citado 2021 jun. 10]; 139(2): 1-8. Disponível em: https://doi.org/10.1590/15163180.2020.0370.R1.18112020

14. Laws MB, Lee Y, Rogers WS, Taubin T, Wilson IB. An instrument to assess HIV related knowledge and adjustment to HIV+ status, and their association with anti-retroviral adherence. PLoS ONE [Internet]. 2020 [citado 2021 jun 9]; 15(6): e0227722. Disponível em: https://doi.org/ 10.1371/journal.pone.0227722

15. Grant-McAuley W, Fogel JM, Galai N, Clarke W, Breaud A, Marzinke MA, et al. Antiretroviral drug use and HIV drug resistance in female sex workers in Tanzania and the Dominican Republic. PLoS ONE [Internet]. 2020 [citado 2021 jun 11]; 15(10): e0240890. Disponível em: https://doi. org/10.1371/journal.pone.0240890

16. Primeira MR, Santos WM, Paula CC, Padoin SM. Qualidade de vida, adesão e indicadores clínicos em pessoas vivendo com HIV. Acta Paul Enferm [Internet]. 2020 [citado 2021 jun 11]; 33: eAPE20190141. Disponível em: http://dx.doi. org/10.37689/ acta-ape/2020AO0141

17. Araújo LL, Teles FML, Karina RR, Carvalho FA, Elucir G, Gimeniz GMT. Adherence to 
antiretrovirals in people coinfected with the human immunodeficiency virus and tuberculosis. Rev Latino Am Enfermagem [Internet]. 2016 [citado 2020 fev 19]; 24: e2691. Disponível em: https:// doi.org/10.1590/1518-8345.0537.2691

18. Rodovalho AG, Tristão FN, Galvão LLC, Rodovalho RG, Torunsky RC, Lucchese R. Associação entre o uso de antirretrovirais no tratamento para HIV e alteraçôes físicas e metabólicas. Blucher Education Proceedings [Internet]. 2017 [citado 2020 mar 9]; 2(1): 15-26. Disponível em: http:// pdf.blucher.com.br.s3-sa-east-1.amazonaws.com/ educationproceedings/sma2016/002.pdf

19. Carvalho PP, Barroso SM, Coelho HC, Penaforte FRO. Fatores associados à adesão à Terapia Antirretroviral em adultos: revisão integrativa de literatura. Ciênc saúde coletiva [Internet]. 2019 [citado 2020 mar 9]; 24(7): 2543-55. Disponível em: https://doi.org/10.1590/141381232018247.22312017

20. Foresto JS, Melo ES, Costa CRB, Antonini M, Gir E, Reis RK. Adesão à terapêutica antirretroviral de pessoas vivendo com HIV/aids em um município do interior paulista. Rev gaúcha enferm [Internet].
2017 [citado 2020 fev 27]; 38(1): e63158. Disponível em: https://doi.org/10.1590/19831447.2017.01.63158

21. Medeiros RCSC, Medeiros JA, Silva TAL, Andrade RD, Medeiros DC, Araújo JS, et al. Qualidade de vida, fatores socioeconômicos, clínicos e prática de exercício físico em pessoas vivendo com HIV/aids. Rev Saúde Pública [Internet]. 2017 [citado 2021 jun 12]; 51(66): 1-8. Disponível em: https://doi. org/10.1590/S1518-8787.2017051006266

22. Bastard M, Fall MB, Lanièce I,Taverne B, Desclaux A, Ecochard R, et al. Revisting Long-term Adherence to Highly Active Antiretroviral Therapy in Senegal Using Latent Class Analysis. J Acquir Immune Defic Syndr [Internet]. 2011 [citado 2020 fev 27]; 57(1): 55-61. Disponível em: https:// www.ncbi.nlm.nih.gov/pubmed/21775934

23. Shefer TA. Resisting the binarism of victim and agent: Critical reflections on 20 years of scholarship on young women and heterosexual practices in South African contexts. Global Public Health [Internet]. 2016 [citado 2020 mar 19]; 11(1-2): 211-223. Disponível em: https://www.tandfonline. com/doi/abs/10.1080/17441692.2015.1029959 